\title{
Innovative Design for Vortex Micro-Nano Bubble generator Based on TRIZ

\author{
Yanjie Chen
}

Chongqing Vocational Institute of Engineering, Jiangjin District, Chongqing, China , 402260

qizheng_c@163.com

\begin{abstract}
Keywords: TRIZ; micro-nano bubble; bubble generating device; bubble generation technology; Vortex; innovative design; bubble generator

Abstract. In order to solve the issues of the micro-nano bubble generation technology, that generally exists high energy consumption and low bubble production, TRIZ method of technical contradiction inventive principles was utilized to provide ideas to design a new-type of micro-nano bubble generating device. The technical contradiction of Sadatomi 's micro-nano bubble generator was described by a pair of general engineering parameters, and the innovative principles were obtained by the technology of contradiction matrix. The concrete implementations of these innovation theories were carried, combing with the actual situation of the micro-nano bubble generation. And a new type of vortex micro-nano bubble generator is developed which shall be more suitable for the bubble generation.
\end{abstract}

\section{Introduction}

Micro-nano bubble refers to the bubble diameter between 0.1 - 50 microns bubble. This bubble possesses the characteristics of large surface area, slow rise, self pressurization dissolution, surface charge, resulting in a large number of free radicals, high mass transfer efficiency and higher characteristic of gas dissolution rate ${ }^{[1]}$. It has a broad application prospect in the field of science and technology interface.

At present, micro bubble generation technology has been widely applied to many fields ${ }^{[2]}$,such as mineral processing, ecological restoration, sewage treatment, water purification technology, ozone water disinfection, bio pharmaceutical, fine chemical reaction ,etc . With the further application of micro-nano bubble technology in life and production, micro-nano bubble generation technology has become an important issue. Therefore, it has important practical application value to carry out the research on the bubble generation technology and the bubble generating device, which can generate micro-nano bubbles efficiently and effectively.

According to the generation mechanism of micro-nano bubbles, micro-nano bubble generation technology mainly includes the methods of dissolved gas, dispersed air, electrolysis, chemical reaction and ultrasonic cavitation, etc. However, conventional micro-bubble generation technology and micro-bubble generating device, which is widespread low efficiency, big power phenomenon and mastered in the hands of foreign developed countries, is not conducive to the popularization and application in actual production. Therefore, the research and development of a innovative type of micro-nano bubble generator, which is higher efficiency and higher adaptability, has the important theory and practical significance.

\section{Problem analysis of Sadatomi 's micro-nano bubble generator}

Sadatomi 's micro-nano bubble generator is a typical bubble generating device. Its structure includes a circular pipe, nozzle, spherical body, suction chamber, micro pore, mixing chamber and a diffusion tube. The front end of the pipeline is provided with a nozzle. The spherical body is arranged in the middle of the pipe. The suction chamber is arranged on the pipeline after the spherical body. And the micro hole is arranged on the air suction chamber pipe. The work process: the liquid from the nozzle is launched into the pipeline. When the liquid flowing through the spherical body, the spherical body forms a negative pressure area, which is behind the sphere. Under the action of negative pressure, gas 
is inhaled into the mixing tube from micro pores on the wall. In the mixing tube, gas is accelerated and dispersed by high-speed movement of the droplet and gas. Then in the diffusion tube section, gas is compressed into micro-namo bubbles.

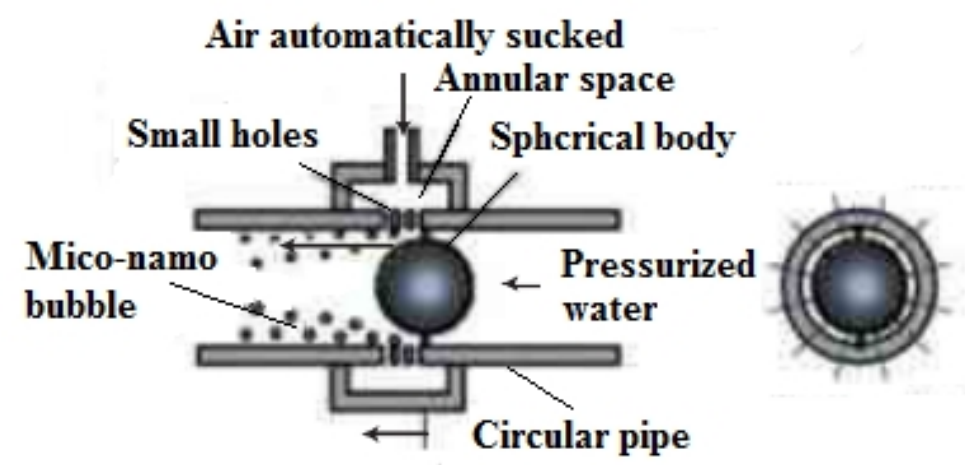

Fig. 1 The illustration of Sadatomi 's micro-nano bubble generator

Qualified micro-nano bubbles can be generated by Sadatomi 's micro-nano bubble generator, but the efficiency of micro bubble formation is low. The size of negative pressure determines the amount of micro-nano bubble generated. The greater the negative pressure, the more the amount of micro bubble generated relatively. And want to get more negative pressure, more small spheres need to be set. But it is difficult to set more spheres in the circular pipe , and to manufacture more small holes. So the main problem of Sadatomi 's micro-nano bubble generator applied to generate micro-nano bubble is how to solve the contradiction between "Higher productivity" and "Greater negative pressure".

\section{Design research of Vortex Micro-Nano Bubble generator based on TRIZ}

A. The Innovative design process based on TRIZ. In TRIZ theory, a specific problem waiting for solving should be transformed into technical contradictions---what is called as standard problem model by 39 general engineering parameters, after that, for this type of problem model, using the problem-solving tool---contradiction matrix to find out the suitable innovative-theory, deducing and materializing finally, the possible options for specific problems will be obtained ${ }^{[3]}$.

\section{B. Technology contradiction analysis of Sadatomi 's micro-nano bubble generator.}

Technology contradiction refers to the mutual restriction relationship of two parameters in the technology system. Briefly, when improving one parameter or character in technology system, the other parameter or character becomes deteriorate, which causes technology contradiction ${ }^{[4]}$. By analyzing the main problem of Sadatomi 's micro-nano bubble generator, there exists a pair of technical contradiction between "Higher productivity" and "Greater negative pressure".

So this study gets the innovative principles recommended by TRIZ through contradiction matrix, then according to these innovative principles as inspiration, it is easy to find some reasonable schemes to solve the problem.

(1) Determination of general engineering parameters. These 39 general engineering parameters can well describe most of technical contents in projects. They transform the contradictions in practical engineering design into the standard technical contradictions. By analyzing the technical contradictions of Sadatomi 's micro-nano bubble generator and using 39 engineering parameters, find that: "Higher productivity" matches with 39\# "productivity", and "Greater negative pressure" matches with 10\# "pressure". That is, 39\# "productivity" is an improved engineering parameter, but the deteriorated engineering parameter is $10 \#$ "pressure".

(2) Application of contradiction matrix. After the research of huge amount of literature by Altshuller, contradiction matrix, as shown in table 1, is established as a tool to analyze and solve technical contradictions. In the contradiction matrix, the first column represents improved engineering parameters, but the first line represents deteriorated ones while improving one parameter in the system. 
Table 1 Contradiction matrix

\begin{tabular}{lcc}
\hline Improve parameters & Deterioration parameters & Inventive principles \\
\hline 39\# "productivity" & 10\# "pressure" & $28 \#, 15 \#, 10 \#, 36 \#$ \\
\hline
\end{tabular}

\section{Innovative design analysis of Vortex Micro-Nano Bubble generator.}

According to innovative principles, it analyze the concrete problems, find some reasonable schemes and choose the best one. Each number of the contradiction matrix denotes the most useful innovative principles by TRIZ to solve the corresponding technical contradictions. Analyzed above based on, four principles of innovation can be obtained, as shown in Table 2.

Table 2 Description of the innovation principles defined

\begin{tabular}{lll}
\hline $\begin{array}{l}\text { Principle } \\
\text { Number }\end{array}$ & Innovation Principle & Description of the innovation principles defined \\
\hline $28 \#$ & $\begin{array}{l}\text { Replacement of } \\
\text { mechanical system }\end{array}$ & $\begin{array}{l}\text { Field substitution; from a constant field to a variable field; from a fixed field to a } \\
\text { time varying field;from the field to the organized field. } \\
\text { Adjust physical or environmental performance, so that in all stages of the work to } \\
\text { the best condition. }\end{array}$ \\
$10 \#$ & Pynamics theory & $\begin{array}{l}\text { To complete some or all of the actions or functions in advance. } \\
\text { An effect or phenomenon that occurs during the transformation of the phase } \\
\text { transition of an object. }\end{array}$ \\
\hline
\end{tabular}

The following is the designs of innovation principles on the basis of technology contradictory of Vortex Micro-Nano Bubble generator.

Structure design: According to 28\# innovative principles, application of vortex type, which instead of a single gravitational field with a density field and a centrifugal field, provides a greater negative pressure area. Under the action of small spheres, which arranged in the circular pipe, a lot of negative pressure areas are formed in each region of the axial direction.

Working process: According to $15 \#, 10 \#$ and 36\# innovative principles, the gas-liquid mixture is firstly formed. Under the combined action of density field, centrifugal force field and negative pressure,the gas is separated from the gas-liquid mixture. Then the gas moves toward the axis,in which process, the bubbles are gradually broken into micro-nano bubbles by small spheres.

\section{E. Innovative design of Vortex Micro-Nano Bubble generator.}

This paper proposes an innovative design of Vortex Micro-Nano Bubble generator by using the innovative principles above.Vortex Micro-Nano Bubble generator is based on the principle of fluid mechanics. The structure of Vortex Micro-Nano Bubble generator is shown in Fig. 2. The main structural components, including: Vortex body, Gas imports, Spherical body, Nozzle,etc. And Vortex body, which is filled by Spherical body, is cylindrical and conical.

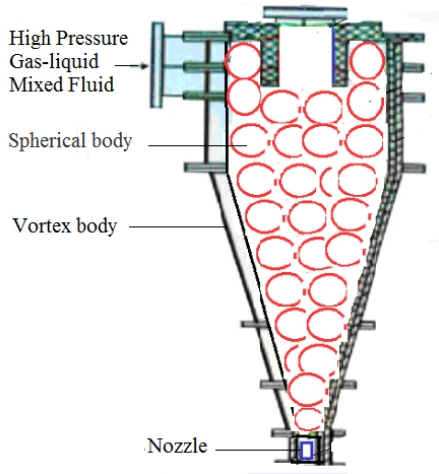

Fig. 2 Schematic diagram of the overall structure of Vortex Micro-Nano Bubble generator

The working principle of Vortex Micro-Nano Bubble generator is shown in Fig. 3. Firstly, High pressure gas-liquid mixed fluid does rotary motion along the swirl spinning motion in the Vortex body, and is gradually accelerated along the direction of the Vortex body cone. In the interaction of fluid density field and centrifugal force field, a negative pressure is generated in the center of the column at the Vortex body. Gases, which are separated from the high pressure gas-liquid mixed fluid, are gradually collected to the negative pressure. While flowing through the surface of the sphere, the 
high pressure gas-liquid mixed fluid is continuously accelerated,and Gases are continuously further cut into minute bubbles by liquid. Meanwhile, the bubbles in the negative pressure moves towards the nozzle. When the bubbles are ejected from the nozzle, the high speed strength shear and the high frequency pressure change are generated at the gas-liquid contact interface, and Micro-Nano Bubble are generated.

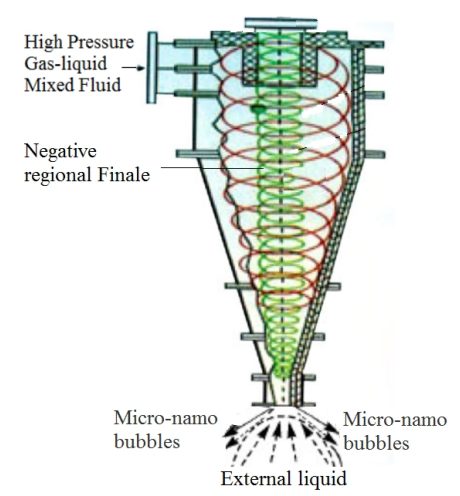

Fig.3 The working principle of Vortex Micro-Nano Bubble generator

\section{Conclusions}

To sum up, the technical contradiction of Sadatomi 's micro-nano bubble generator was described by a pair of general engineering parameters, and the innovative principles were obtained by the technology of contradiction matrix. The concrete implementations of these innovation theories were carried, combing with the actual situation of the micro-nano bubble generation. And a new type of vortex micro-nano bubble generator is developed which shall be more suitable for the bubble generation.

\section{References}

[1] Takahashi M, Kawamura T,Yamamoto Y, etal. Effect of Shrinking Microbubble on Gas Hydrate Formation. J Physchem B, Forum Vol. 107( 2003), p. 2171

[2] Liu S H,Wang Q, etal. Effect of micro-bubbles on coagulation flotation process of dyeing wastewater. Separation and Purification Technology,Forum Vol. 71( 2010), p.337.

[3] Min Zhao, Xiaoling Shi, Haibo Duan, in: Triz Introduction and Practice, edited by Science Press, China, Beijing(2009), in press.

[4] Peibo Tan, Haiyan Ru, in: The Innovation Algorithm, edited by Huazhong University of Science \& Technology Press, China,Wuhan(2008),in press. 